\title{
Feynman Integrals and Scattering Amplitudes from Wilson Loops
}

\author{
Song He, ${ }^{1,2,3,4, *}$ Zhenjie Li® ${ }^{1,4, \dagger}$ Qinglin Yang $\oplus^{1,4, \dagger}$ and Chi Zhang $\oplus^{5, \S}$ \\ ${ }^{1}$ CAS Key Laboratory of Theoretical Physics, Institute of Theoretical Physics, Chinese Academy of Sciences, \\ Beijing 100190, China \\ ${ }^{2}$ School of Fundamental Physics and Mathematical Sciences, Hangzhou Institute for Advanced Study, \\ UCAS, Hangzhou 310024, China \\ ${ }^{3}$ ICTP-AP International Centre for Theoretical Physics Asia-Pacific, Beijing/Hangzhou, China \\ ${ }^{4}$ School of Physical Sciences, University of Chinese Academy of Sciences, \\ No.19A Yuquan Road, Beijing 100049, China \\ ${ }^{5}$ Niels Bohr International Academy, Niels Bohr Institute, Copenhagen University, \\ Blegdamsvej 17, 2100 Copenhagen $\emptyset$, Denmark
}

(Received 8 March 2021; accepted 18 May 2021; published 9 June 2021; corrected 7 July 2021)

\begin{abstract}
We study Feynman integrals and scattering amplitudes in $\mathcal{N}=4$ super-Yang-Mills theory by exploiting the duality with null polygonal Wilson loops. As the main application, we compute for the first time the symbols of the general double pentagon integrals, which give the finite part of two-loop maximally helicity violating (MHV) amplitudes and finite components of next-to-MHV (NMHV) amplitudes to all multiplicities. The rational parts of the symbol consist of 164 letters, while the algebraic part contains 96 algebraic letters and cancel in MHV amplitudes and NMHV components which are free of square roots.
\end{abstract}

DOI: 10.1103/PhysRevLett.126.231601

Introduction.-Scattering amplitudes are central objects in fundamental physics: they are crucial for connecting theory to experiments in particle accelerators such as the Large Hadron Collider, and they play a central role in discovering new structures of quantum field theory (QFT). As arguably the simplest QFT, tremendous progress has been made for planar $\mathcal{N}=4$ supersymmetric Yang-Mills theory (SYM); not only have hidden mathematical structures for all-loop integrands been unraveled [1-3], but the integrated amplitudes have also been computed to impressively high loops, for $n=6,7$ [4] and for higher multiplicities [5-8]. Moreover, these studies have made $\mathcal{N}=4$ SYM an extremely fruitful playground for new methods of evaluating Feynman integrals, which is a subject of enormous interest (cf. [9-11] and references therein).

In planar $\mathcal{N}=4 \mathrm{SYM}$, a remarkable duality between maximally helicity violating (MHV) scattering amplitudes and null polygonal Wilson loops (WL) was discovered at both strong [12] and weak coupling [13,14]; later it was established that superamplitudes (after stripping off MHV tree prefactor) are dual to supersymmetric WL $[15,16]$, and quite a lot of what we have learned about amplitudes are from this dual picture. Based on integrability [17] and operator product expansions of WL [18], one can compute amplitudes

Published by the American Physical Society under the terms of the Creative Commons Attribution 4.0 International license. Further distribution of this work must maintain attribution to the author(s) and the published article's title, journal citation, and DOI. Funded by SCOAP . at any value of the coupling around collinear limits [19]; the powerful $\bar{Q}$ anomaly equation [20] for computing multiloop amplitudes $[7,8]$ was derived from the dual WL as well. In this Letter, we exploit the dual picture in yet another context: the computation of certain Feynman integrals [21].

Recall that in computing (super-)WL, one inserts fields in the supermultiplet at edges and vertices of the null polygon, as well as chiral Lagrangians at dual points which correspond to loop variables to be integrated over [15]. We will see that, certain loop integrals for scattering amplitudes are more easily performed as Feynman diagrams of WL where (some) loop insertions can be trivially integrated out and yield relatively simple integrations over edge insertions (and remaining loops). In this way, we obtain the " $d \log$ " representation for loop integrals and amplitudes made of them [22], which not only makes the evaluation much simpler, but also various desired properties manifest. We initiate the systematic study of $d \log$ representations for a wide range of simple loop integrals in [23], but here we focus on the computation of a class of particularly important integrals, the double pentagons [24]. We denote such an integral as $I_{\mathrm{dp}}(i, j, k, l)$ with massless corners $i, j, k, l$ (which is finite for $j>i+1$ and $l>k+1$ ):

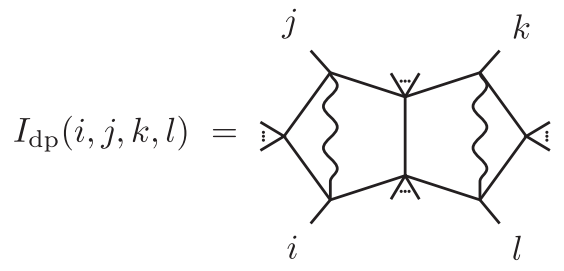




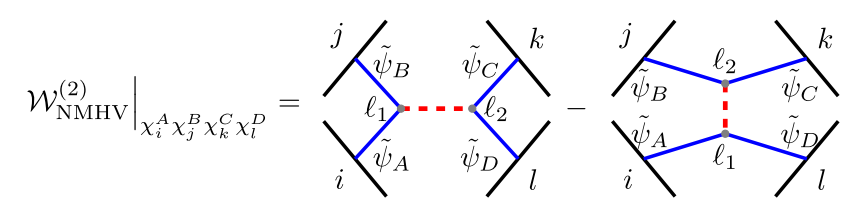

FIG. 1. NMHV component of super-WL as difference of two diagrams, each equal to a double-pentagon integral.

Remarkably, the two-loop MHV amplitudes are given by the sum of $I_{\mathrm{dp}}(i, j, k, l)$ with $i<j<k<l<i$ cyclically (including divergent boundary terms) [24], and these integrals also give a large class of components of twoloop NMHV amplitudes. This fact can be derived from the local-integral representation of amplitudes [24]; let us review pictorially how $I_{\mathrm{dp}}$ 's naturally give NMHV components of supersymmetric WL. Recall that the polygonal WL are most nicely formulated in terms of momentum twistors [25], which correspond to null rays in the dual spacetime and manifest the $\operatorname{SL}(2,2)$ dual conformal symmetries [26]: the vertices $x_{i}$ are given by $\left(x_{i+1}-x_{i}\right)^{\alpha \dot{\alpha}}=\lambda_{i}^{\alpha} \tilde{\lambda}_{i}^{\dot{\alpha}}$, and similarly for the Grassmann part $\left(\theta_{i+1}-\theta_{i}\right)^{\alpha I}=\lambda_{i}^{\alpha} \eta_{i}^{I}$. Then, we introduce the (super) momentum twistors $\mathcal{Z}_{i}=\left(Z_{i}^{a} \mid \chi_{i}^{A}\right):=\left(\lambda_{i}^{\alpha}, x_{i}^{\alpha \dot{\alpha}} \lambda_{i \alpha} \mid \theta_{i}^{\alpha A} \lambda_{i \alpha}\right)$, and hence the Plücker coordinates $\langle i j k l\rangle=$ $\epsilon_{a b c d} Z_{i}^{a} Z_{j}^{b} Z_{k}^{c} Z_{l}^{d}$ with the standard Levi-Civita symbol $\epsilon_{a b c d}$. Consider the component $\chi_{i}^{A} \chi_{j}^{B} \chi_{k}^{C} \chi_{l}^{D}$ of NMHV super-WL, with nonadjacent $i<j<k<l$. It is easy to see that such a component is given by the difference of two Feynman diagrams of WL (Fig. 1.).

To see that each diagram exactly gives a double pentagon, we refer to the argument in [15]: after integrating out fermion insertions along the edges, we obtain all propagators and the numerators ("wavy lines") of $I_{\mathrm{dp}}$, with loop integrations over the insertion points $\ell_{1}, \ell_{2}$. Thus from the WL picture alone, we see that the simplest NMHV component amplitudes at two loops are given by a difference of two WL diagrams, $I_{\mathrm{dp}}(i, j, k, l)-I_{\mathrm{dp}}(j, k, l, i)$.

The double pentagon $I_{\mathrm{dp}}$ has only been evaluated for $n \leq 7$ legs [10,27] [28]. Starting $n=8$, this integral generically depends on functions of kinematics that contain irreducible square roots of Gram determinants, which we call "algebraic letters" [10]. The most general $I_{\mathrm{dp}}(i, j, k, l)$ depends on 12 legs, $i-1, i, i+1, \ldots, l-1, l, l+1$, which has an identical kinematic space as that of the chiral octagon [24]; the generic $I_{\mathrm{dp}}$ is expected to contain 16 square roots corresponding to 16 four-mass box configurations of the latter, and similarly for all finite degenerations. Its analytic computation is currently beyond the reach of conventional method, e.g., Feynman parametrization. The up-to-date result is the numeric computation of $I_{\mathrm{dp}}(1,3,5,7)$ with $n=8$ at a particular kinematic point [29], which suggests that $I_{\mathrm{dp}}(i, j, k, l)-I_{\mathrm{dp}}(j, k, l, i)$ is free of square roots. This surprising observation has been confirmed by an independent $\bar{Q}$ calculation for two-loop NMHV amplitudes [7,8], which shows that the above components are free of square roots for any $n$. However, the $\bar{Q}$ equation is for the full amplitude, thus has no access to the individual $I_{\mathrm{dp}}$ involving algebraic letters. In this Letter, we solve this long-standing problem by evaluating the symbol [30] of the most generic $I_{\mathrm{dp}}(i, j, k, l)$ with $n \geq 12$ from WL. This amounts to the first all-multiplicity computation of all finite integrals for two-loop MHV amplitudes and these special components of NMHV amplitudes.

The key lies in the fact that we can swap the order of integrations in WL diagrams: For $I_{\mathrm{dp}}$, it is possible to perform both loop integrations and be left with fourfold integrals over edge insertions, but in practice a mixture of integrations turns out to be more convenient. We apply the trick only for one of the loop integrations and evaluate the other one by the usual box expansion [31]. In this way, we express $I_{\mathrm{dp}}$ as a sum of twofold $d \log$ integrals of some polylogarithms of weight 2 , which turn out to be similar to $\bar{Q}$ computations $[7,8,20]$ and the predecessor [6]. An important technical point is that when performing the twofold integrations, one needs to "rationalize" square roots in four-mass box integrals. Among other things, we find remarkably compact "algebraic words" of the symbol containing 16 square roots, where for each of them, only 4 new algebraic letters appear compared with the corresponding four-mass box. We see how these algebraic words nicely cancel in the difference for NMHV components, as well as in the cyclic sum for MHV amplitudes. Even more remarkably, the complete symbol for generic $I_{\mathrm{dp}}(i, j, k, l)$ can be expressed compactly using two independent weight-3 integrable symbols, which we will present explicitly. The alphabet contains 164 rational letters, in addition to the algebraic ones.

Warmup example: Chiral pentagon.-Before moving to $I_{\mathrm{dp}}$, let us illustrate this method using the one-loop chiral pentagon (Fig. 2), which is the ingredient of one-loop MHV amplitudes. The integral has four propagators associated with $i, j$, and the last one specified by a generic line $L$ :

$$
I_{\mathrm{p}}(i, j, L):=\int \frac{d^{4} \ell\langle\ell \bar{i} \cap \bar{j}\rangle\langle L i j\rangle}{\langle\langle\ell i\rangle\rangle\langle\langle\ell j\rangle\langle\ell L L},
$$

where the loop momentum $\ell$ can be understood as a point in dual space, and hence a line in twistor space. Here we introduce a shorthand $\langle\langle\ell i\rangle\rangle:=\langle\ell i-1 i\rangle\langle\ell i i+1\rangle$. The numerator depends on the two solutions of the twomass-easy Schubert problem: $(i j)$ and the intersection of planes $\bar{i}:=\epsilon_{a b c d} Z_{i-1}^{b} Z_{i}^{c} Z_{i+1}^{d}$ and $\bar{j}$ [32]. 


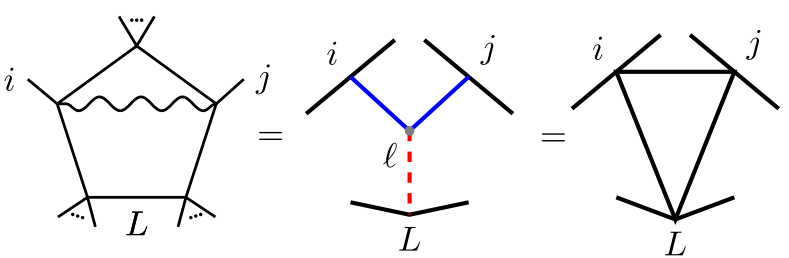

FIG. 2. The chiral pentagon written as a WL diagram, and loop integral performed using "star-triangle" identity.

In [15], $I_{\mathrm{p}}$ was interpreted as a (bosonic) WL diagram with gluons inserted at edge $i, j$ and a Lagrangian insertion at $\ell$. For our purpose, it is convenient to represent $I_{\mathrm{p}} /\langle L i j\rangle$ as a WL diagram with two fermions inserted at edge $i, j$, both connected to the Yukawa vertex $\psi \psi \psi \phi(\ell)$, and a scalar propagator from $\ell$ to the reference line $L$ (Fig 2). To see this, we write $\langle\langle\ell i\rangle\rangle^{-1}$ as $1 \mathrm{D}$ integral $\int_{0}^{\infty}\langle\ell i X(\tau)\rangle^{-2} d \tau$, where we have introduced the twistor interpolating between $Z_{i-1}$ and $Z_{i+1}$ [15]: $X(\tau):=Z_{i-1}+\tau Z_{i+1}$. Note $x:=(i X)$ corresponds to the insertion point on edge $i$ (with two endpoints given by $\tau \rightarrow 0, \infty)$ and similarly for $y:=(j Y)$; the numerator is obtained by taking into account that for fermion propagators $[i|(x-\ell)(\ell-y)| j] \propto\langle\ell \bar{i} \cap \bar{j}\rangle$. Remarkably, one can easily perform the loop integration for this diagram

$\int \frac{d^{4} \ell d^{2} \tau\langle\ell \bar{i} \cap \bar{j}\rangle}{\langle\ell i X\rangle^{2}\langle\ell j Y\rangle^{2}\langle\ell L\rangle}=\int_{0}^{\infty} \frac{d^{2} \tau\langle L \bar{i} \cap \bar{j}\rangle}{\langle L i X\rangle\langle L j Y\rangle\langle i X j Y\rangle}$,

where we have used a version of star-triangle identity (Fig. 2) for three-point functions in conformal field theory [33]. By (2), one can represent $I_{\mathrm{p}}(i, j, L)$ as a twofold line integration over two $d$ log's,

$$
I_{\mathrm{p}}(i, j, L)=\int d \log \frac{\langle L j Y\rangle}{\langle\bar{i}(j Y) \cap(i L)\rangle} d \log \frac{\langle i X j Y\rangle}{\langle L i X\rangle},
$$

where the integration domain for $X, Y$ are edge $i$ and $j$. This expression makes it clear that it is a pure function, that is a linear combination of polylogarithms with numerical coefficients, of weight 2 . In this form, the integration is trivial and yields the well-known result; this WL representation not only trivializes the evaluation of integrals, but also manifests properties of the answer, such as dual conformal invariance (DCI) and uniform transcendental weights.

The double pentagon as twofold integrals.-Now we turn to the main object of interests: the double pentagon integral,

$I_{\mathrm{dp}}(i, j, k, l):=\int \frac{d^{4} \ell_{1} d^{4} \ell_{2}\left\langle\ell_{1} \bar{i} \cap \bar{j}\right\rangle\left\langle\ell_{2} \bar{k} \cap \bar{l}\right\rangle\langle i j k l\rangle}{\left.\left\langle\left\langle\ell_{1} i\right\rangle\right\rangle\left\langle\ell_{1} j\right\rangle\right\rangle\left\langle\ell_{1} \ell_{2}\right\rangle\left\langle\left\langle\ell_{2} k\right\rangle\right\rangle\left\langle\left\langle\ell_{2} l\right\rangle\right\rangle}$.
Without explicitly using the WL diagram, we apply the same manipulation as above for loop $\ell_{1}$ to write it as an integration of a one-loop hexagon over edge $i, j$ :

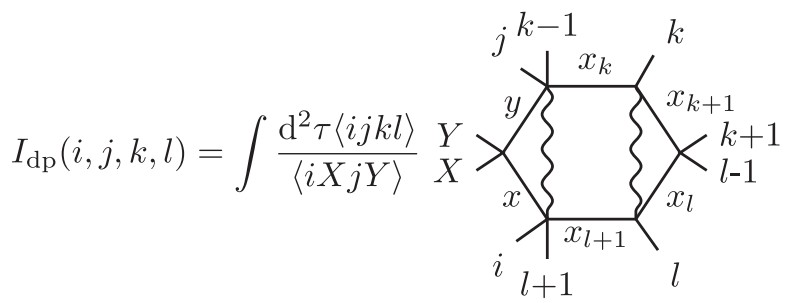

where the one-loop hexagon is defined as

$$
I_{\text {hex }}:=\int \frac{d^{4} \ell_{2}\left\langle\ell_{2} \bar{i} \cap \bar{j}\right\rangle\left\langle\ell_{2} \bar{k} \cap \bar{l}\right\rangle}{\left\langle\ell_{2} i X\right\rangle\left\langle\ell_{2} j Y\right\rangle\left\langle\left\langle\ell_{2} k\right\rangle\right\rangle\left\langle\left\langle\ell_{2} l\right\rangle\right\rangle} .
$$

Note that it has two "deformed" legs $X, Y$ rather than original $i+1$ and $j-1$. The computation of $I_{\text {hex }}$ is standard - using the familiar box expansion or the general algorithm provided in [34]. Either way, the result turns out to be a linear combination of $\left(\begin{array}{l}6 \\ 4\end{array}\right)=15$ box integrals with some $d \log 2$-forms as the coefficients.

To describe our result, it is convenient to label the six propagators of $I_{\text {hex }}$ by the six points $x, y, x_{k}, x_{k+1}, x_{l}, x_{l+1}$ in the dual spacetime [as in Eq. (5)] and introduce the " $\gamma$ "-deformed four-mass box function: $\tilde{F}:=\gamma F(u, v)-1 / 2 \log u \log v$, where we have introduced $\gamma:=r_{1}-r_{2} / r_{1}+r_{2}$ and

$$
F(u, v):=\mathrm{Li}_{2}(1-z)-\mathrm{Li}_{2}(1-\bar{z})+\frac{1}{2} \log \left(\frac{z}{\bar{z}}\right) \log (v)
$$$$
\text { with } \quad u=u_{a, b, c, d}=z \bar{z}, \quad v=u_{b, c, d, a}=(1-z)(1-\bar{z}) \text {, }
$$

and $u_{a, b, c, d}:=x_{a, b}^{2} x_{c, d}^{2} /\left(x_{a, c}^{2} x_{b, d}^{2}\right), r_{1}$ and $r_{2}$ are leading singularities [24] evaluated at two solutions of the fourmass Schubert problem. Then, $I_{\mathrm{dp}}$ can be compactly expressed as

$$
\int\left(\left[x, x_{k}\right] I_{x, x_{k}}-(k-1 \leftrightarrow k+1)\right)-(\bar{k} \leftrightarrow \bar{l})+[x, y] I_{x, y}
$$

where the second term is obtained by swapping $k-1$ with $k+1,(\bar{k} \leftrightarrow \bar{l})$ denotes terms given by swapping $k, x_{k}$ or $x_{k+1}$ with $l, x_{l}$ or $x_{l+1}$ for the first two terms [35], and 


$$
\begin{aligned}
{[x, y]=} & d \log \frac{\langle i X k l\rangle}{\langle i X j Y\rangle} d \log \frac{\langle\bar{i}(j Y) \cap(i k l)\rangle}{\langle j Y k l\rangle}, \\
{\left[x, x_{k}\right]=} & d \log \frac{\langle j Y i l\rangle}{\langle j Y k l\rangle} d \log \frac{\langle i X j Y\rangle}{\langle l(i X)(j Y)(k k+1)\rangle}, \\
I_{x, x_{k}}:= & \tilde{F}\left(x, y, x_{k+1}, x_{l}\right)-\tilde{F}\left(x, y, x_{k+1}, x_{l+1}\right) \\
& -\mathrm{L}_{2}(l+1, x, y, l)+\mathrm{L}_{2}(l+1, x, k+1, l) \\
& -\mathrm{L}_{2}(l+1, y, k+1, l)+\log u_{l+1, x, y, l} \log u_{x, y, k+1, l+1}, \\
I_{x, y}:= & \mathrm{L}_{2}(x, k, k+1, l)-\mathrm{L}_{2}(x, k, k+1, l+1) \\
& -\mathrm{L}_{2}(l+1, x, k, l)+\mathrm{L}_{2}(l+1, x, k+1, l) \\
& -\mathrm{L}_{2}(l+1, k, k+1, l)+\log u_{l+1, x, k, l} \log u_{x, k, k+1, l+1}
\end{aligned}
$$

with $\mathrm{L}_{2}(a, b, c, d):=\mathrm{Li}_{2}\left(1-u_{a, b, c, d}\right)$. We remark that (7) has a number of desirable properties. It is manifestly DCI and expected to yield weight-4 polylogarithms, and one can check that it remains finite even for special cases such as $j=k+1$ or $i=l+1$. Moreover, for the generic case we have 4 four-mass boxes involved, which depend on square roots $\Delta(x, y, k, l):=\sqrt{(1-u-v)^{2}-4 u v}$ (where $u, v$ are defined as above for these four points) etc., and after integrating over $x, y$, each needs to be evaluated at endpoints $x=x_{i}, x_{i+1}$ (similarly for $y$ ). Thus the result must contain the 16 square roots $\Delta(i, j, k, l)$, $\Delta(i+1, j, k, l), \Delta(i, j+1, k, l), \Delta(i+1, j+1, k, l)$ etc. as expected.

Rationalization: Uniform transcendentality, algebraic words and their cancellation.- - Had there been no square root in $I_{\text {hex }}$, it would have been straightforward to perform the twofold integrations in (5). In addition to square roots in $\tilde{F}$ 's, what is worse is the presence of $\gamma$ 's which makes it even obscure that the answer must be pure. It turns out that these issues are resolved by "rationalizing" the square roots, which have been exploited in the $\bar{Q}$ calculation $[7,8]$. The idea is very simple: we make a change of variables such that there is no square root in $I_{\text {hex }}$, then the integral can be performed e.g., at the symbol level using the algorithm given in [20], and square roots only appear via integration domains. Let us consider any of the 4 fourmass boxes $\tilde{F}\left(x(\tau), y\left(\tau^{\prime}\right), *, *\right)$, where the square roots are contained in $z\left(\tau, \tau^{\prime}\right)$ and $\bar{z}\left(\tau, \tau^{\prime}\right)$. We make change of variable from $\tau$ to $z(\tau)$ (suppressing the dependence on $\tau^{\prime}$ ). As for the $\bar{z}$, note that there exist $a$ and $b$, which depend on kinematics and $\tau^{\prime}$, but they are independent of $\tau$, such that $a u(\tau)+b v(\tau)=1$. This allows us to relate $\bar{z}(\tau)$ to $z(\tau)$ by a Möbius transformation $\bar{z}=\Lambda(z):=[b z+(1-b)] /[(a+b) z-b]$.

Something remarkable happens at this stage: the prefactor $\gamma$, together with $d \log$ forms depending on $\tau$, becomes a beautiful $d \log$ of a rational function of $z(\tau)$. The $\tau$ integral for a four-mass box function becomes

$$
\begin{aligned}
& \int_{z(0)}^{z(\infty)} d \log \frac{z-w}{z-\Lambda(w)}\left(\operatorname{Li}_{2}(1-z)-\mathrm{Li}_{2}[1-\Lambda(z)]\right. \\
& \left.\quad+\frac{1}{2} \log \frac{z}{\Lambda(z)} \log \{(1-z)[1-\Lambda(z)]\}\right),
\end{aligned}
$$

for some $w$ and $\Lambda(w)$, both of which are independent of $\tau$. At this stage, it becomes obvious that $I_{\mathrm{dp}}$ is represented as twofold $d \log$ integrals of weight- 2 pure functions.

In this form, one can perform the $\tau$ integration directly, and it suffices to give the part of the symbol only involving square roots. The algebraic part of the above integral (8) gives a beautiful weight-3 "algebraic word" (of the $\tau^{\prime}$ integrand):

$$
\left.\frac{1}{4}\left(u \otimes \frac{1-\bar{z}}{1-z}+v \otimes \frac{z}{\bar{z}}\right) \otimes \frac{(z-w)[\Lambda(z)-\Lambda(w)]}{(\Lambda(z)-w)[z-\Lambda(w)]}\right|_{\tau=0} ^{\tau=\infty},
$$

where we evaluate the symbol at $\tau=\infty$, minus that at $\tau=0$, which results in square roots $\Delta\left(x_{i}, y, *, *\right)$ and $\Delta\left(x_{i+1}, y, *, *\right)$. Note that the first two entries are exactly the symbol of the four-mass box, $F(u, v)$. One can easily check that these weight- 3 algebraic words cancel in the difference $I_{\mathrm{dp}}(i, j, k, l)-I_{\mathrm{dp}}(j, k, l, i)$.

Next we need to rationalize the square roots in $\tau^{\prime}$ of the above algebraic words to perform the $\tau^{\prime}$ integration. We emphasize a major difference between this step and the previous one from weight 2 to 3 : the $d \log$ factors are manifestly rational due to the absence of a $\gamma$ factor, thus after we change variable from $\tau^{\prime}$ to $z\left(\tau^{\prime}\right)$, the arguments of $d \log$ 's are given by products of the form $(z-w)[z-$ $\left.\Lambda^{\prime}(w)\right]$ rather than ratios. The immediate consequence is that the last entries of the resulting symbol are free of any square roots [36]. In the end, we obtain a remarkably compact expression for algebraic words of the final answer: the first two entries are given by (the symbol of) four-mass boxes, the third entry given by algebraic letters, and the last entry rational ones. Since there are 16 square roots, $\Delta(a, b, c, d)$ for $a:=i+\sigma_{1}, b:=j+\sigma_{2}, c:=k+\sigma_{3}, d:=$ $l+\sigma_{4}$ with $\sigma=0,1$, the algebraic part of the symbol of $I_{\mathrm{dp}}(i, j, k, l)$ can be written as an alternating sum of 16 terms:

$\sum_{\sigma_{a} \in\{0,1\}}(-)^{\sum \sigma} S\left[F\left(i+\sigma_{1}, j+\sigma_{2}, k+\sigma_{3}, l+\sigma_{4}\right)\right] \otimes W_{\sigma_{1}, \ldots, \sigma_{4}}^{i, j, k, l}$

where each term is characterized by a four-mass box $F(a, b, c, d)$; it is accompanied by the last two entries denoted as $W_{\sigma_{1}, \ldots, \sigma_{4}}^{i, j, k, l}$, which contains the same square root $\Delta(a, b, c, d)$ and depends on $x_{a}, \ldots, x_{d}$ and $i, j, k, l$. 


$$
\begin{aligned}
W_{a-i, \ldots, d-l}^{i, j, k, l}= & \chi_{a, b, c, d}^{j, k} \otimes \frac{\left\langle x_{a} j k\right\rangle\left\langle x_{b} i l\right\rangle}{\left\langle x_{a} j l\right\rangle\left\langle x_{b} i k\right\rangle}+\text { cyclic } \\
& +\frac{1}{2}\left(\frac{\bar{z}(1-z)}{z(1-\bar{z})} \prod \chi\right) \\
& \otimes \frac{\left\langle x_{a} j l\right\rangle\left\langle x_{b} i k\right\rangle\left\langle x_{c} j l\right\rangle\left\langle x_{d} i k\right\rangle}{\left\langle x_{a} k l\right\rangle\left\langle x_{b} i l\right\rangle\left\langle x_{c} i j\right\rangle\left\langle x_{d} j k\right\rangle}
\end{aligned}
$$

where the first four terms are given by cyclic rotations of $i$, $j, k, l$ (thus also of $a, b, c, d$ ), and in the last term, both $[\bar{z}(1-z)] /[z(1-\bar{z})]$ and the product $\prod \chi$ are cyclic invariant; the four new algebraic letters are given by

$$
\chi_{a, b, c, d}^{j, k}:=\left(\frac{\frac{\left\langle x_{a} x_{b}\right\rangle\left\langle x_{d} j k\right\rangle}{\left\langle\frac{\left\langle x_{d} x_{b}\right\rangle\left\langle x_{a} j k\right\rangle}{\left\langle x_{a} x_{b}\right\rangle\left\langle x_{d} j k\right\rangle}-z_{a, b, c, d}\right.}}{\frac{\left\langle x_{d} x_{b}\right\rangle\left\langle x_{a} j k\right\rangle}{\langle a, b, c, d}}\right)
$$

and cyclic images $\chi_{b, c, d, a}^{k, l}, \chi_{c, d, a, b}^{l, i}$ and $\chi_{d, a, b, c}^{i, j}$. Note that the algebraic letters are special multiplicative combinations of those found for two-loop NMHV amplitudes in [8].

The way we present $W$ makes manifest a nice symmetry, $W_{\sigma_{1}, \sigma_{2}, \sigma_{3}, \sigma_{4}}^{i, j, k, l}=W_{\sigma_{2}, \sigma_{3}, \sigma_{4}, \sigma_{1}}^{j, k}$, which guarantees that all square roots cancel in $I_{\mathrm{dp}}(i, j, k, l)-I_{\mathrm{dp}}(j, k, l, i)$, as we have seen at the level of weight-3 integrands. It is even more interesting to see how square roots also drop out for two-loop MHV amplitudes (given by a cyclic sum of all $I_{\mathrm{dp}}$ 's). To see this, we collect algebraic words for a given square root: it is easy to see that $16 I_{\mathrm{dp}}$ 's contribute, and the result is given by the tensor product of $S\left[F\left(x_{a}, \ldots, x_{d}\right)\right]$ and the combination

$$
\sum_{\sigma_{a} \in\{0,1\}} W_{\sigma_{1}, \ldots, \sigma_{4}}^{a-\sigma_{1}, \ldots, d-\sigma_{4}}\left(x_{a}, x_{b}, x_{c}, x_{d}\right)
$$

This combination vanishes and hence guarantees the absence of square roots from two-loop MHV amplitudes.

Final results and checks. - In addition to the algebraic part, we also compute the remaining part that is free of any square roots; the computation of the symbol can be done trivially, as long as we apply the integration rule consistently to the complete weight-3 symbol including the algebraic words and the rest [37]. We record the symbol for $I_{\mathrm{dp}}(1,4,7,10)$ with $n=12$ in [38]. Remarkably, we find that the complete symbol can be written in a compact form by organizing it using $8+16$ combinations of the last entries with manifest symmetries. Equivalently, we express its total differential $d I_{\mathrm{dp}}(i, j, k, l)$ as

$$
\begin{gathered}
\frac{1}{2} R_{j-1 j}^{\bar{i}} d \log \frac{\langle i(i-1 i+1)(j-1 j)(k l)\rangle}{\langle\bar{i} j\rangle\langle j-1 j k l\rangle}+M_{j-1 j}^{i k l} d \log \frac{\langle i j-1 j k\rangle}{\langle j-1 j k l\rangle} \\
-(j-1 j \leftrightarrow j j+1)+(\bar{i} \leftrightarrow \bar{j})+(\bar{k} \leftrightarrow \bar{l})+(i j \leftrightarrow k l)
\end{gathered}
$$

where only two independent weight-3 DCI functions, $R_{j-1 j}^{\bar{i}}$ and $M_{j-1 j}^{i k l}$ are needed; each relabeling applies to all previous terms, giving 8 and 16 images of these functions, respectively. The algebraic words (10) contribute to the symbol of $M$ only, while the symbol of $R$ is rational. We present both symbols in [38], where one can easily count out the 164 rational letters of the alphabet.

We have performed thorough checks on our result, such as the physical first entry conditions and first two entries conditions which are manifest in terms of $R_{j-1 j}^{\bar{i}}$ and $M_{j-1 j}^{i k l}$. One can easily check that the symbol is DCI, and as shown in (12) it is symmetric in exchanging $(i, j)$ with $(l, k)$ and in simultaneous exchange $i \leftrightarrow j$ and $k \leftrightarrow l$, as well as antisymmetric under $i-1 \leftrightarrow i+1$ etc. A nontrivial check is to see that the complete symbol is integrable. Moreover, given the most generic $I_{\mathrm{dp}}(i, j, k, l)$, it is important that any finite degeneration of the integral remains well defined. This happens when $j=i+2$ (similarly $l=k+2$ ), or $k=$ $j+2$ (similarly between $l$ and $i$ ), and they can be viewed as (multiple) collinear limits of the original integral. We have checked that in all these cases the symbol remains finite, which also gives results for these special cases. For example, $I_{\mathrm{dp}}(1,3,5,7)$ for $n=8$ can be obtained from the generic case by taking four collinear limits; the symbol, recorded in [38] as well, contains two square roots and 108 rational letters. Last but not least, we take the difference $I_{\mathrm{dp}}(1,3,5,7)-I_{\mathrm{dp}}(3,5,7,1)$ and find perfect agreement with the component $\chi_{1} \chi_{3} \chi_{5} \chi_{7}$ of the 8-point NMHV amplitude from the $\bar{Q}$ calculation [7].

Conclusions and outlook.-We have computed the symbol of all finite double-pentagon integrals $I_{\mathrm{dp}}(i, j, k, l)$ (with $j>i+1$ and $l>k+1$ ), which also amount to all-multiplicity, Feynman-integral computation of the finite part of two-loop MHV amplitudes, and all $\chi_{i} \chi_{j} \chi_{k} \chi_{l}$ components with nonadjacent $i, j, k, l$ of two-loop NMHV amplitudes. The alphabet consists of 96 algebraic letters ( 6 for each of the 16 square roots), and 164 rational letters. We see not only desirable physical conditions on the first two entries, but also more interesting patterns for the complete symbol. The compact expression (12) with the symbols of $R$ and $M$ in [38] deserves further investigation, which also give a compact formula for the square-root-free symbol of two-loop NMHV components. It would be 
interesting to determine the weight-3 functions $R$ and $M$, which may have interesting physical meaning themselves. Of course, it would also be nice to upgrade the symbol to weight 4 functions (one possibility being the bootstrap method along the lines of [10]).

We would like to thank Nima Arkani-Hamed, Yichao Tang, and Yang Zhang for inspiring discussions. The research of S.H. is supported in part by the Key Research Program of Frontier Sciences of CAS under Grant No. QYZDBSSW-SYS014, Peng Huanwu Center under Grant No. 12047503, and National Natural Science Foundation of China under Grant No. 11935013. C. Z. was supported in part by the ERC starting Grant No. 757978 and Grant No. 00025445 from the Villum Fonden.

*songhe@itp.ac.cn

†lizhenjie@itp.ac.cn

*yangqinglin@itp.ac.cn

§chi.zhang@nbi.ku.dk

[1] N. Arkani-Hamed, J. L. Bourjaily, F. Cachazo, S. CaronHuot, and J. Trnka, J. High Energy Phys. 01 (2011) 041.

[2] N. Arkani-Hamed, J. L. Bourjaily, F. Cachazo, A. B. Goncharov, A. Postnikov, and J. Trnka, Grassmannian Geometry of Scattering Amplitudes (Cambridge University Press, Cambridge, England, 2016).

[3] N. Arkani-Hamed and J. Trnka, J. High Energy Phys. 10 (2014) 030.

[4] L. J. Dixon, J. M. Drummond, and J. M. Henn, J. High Energy Phys. 11 (2011) 023; L. J. Dixon, J. M. Drummond, C. Duhr, M. von Hippel, and J. Pennington, Proc. Sci., LL2014 (2014) 077 [arXiv:1407.4724]; L. J. Dixon and M. von Hippel, J. High Energy Phys. 10 (2014) 065; J. M. Drummond, G. Papathanasiou, and M. Spradlin, J. High Energy Phys. 03 (2015) 072; L. J. Dixon, M. von Hippel, and A. J. McLeod, J. High Energy Phys. 01 (2016) 053; S. CaronHuot, L. J. Dixon, A. McLeod, and M. von Hippel, Phys. Rev. Lett. 117, 241601 (2016); L. J. Dixon, J. Drummond, T. Harrington, A. J. McLeod, G. Papathanasiou, and M. Spradlin, J. High Energy Phys. 02 (2017) 137; J. Drummond, J. Foster, Ö. Gürdoğan, and G. Papathanasiou, J. High Energy Phys. 03 (2019) 087; S. Caron-Huot, L. J. Dixon, F. Dulat, M. von Hippel, A. J. McLeod, and G. Papathanasiou, J. High Energy Phys. 08 (2019) 016; 09 (2019) 061; L. J. Dixon and Y.-T. Liu, J. High Energy Phys. 10 (2020) 031.

[5] C. Anastasiou, A. Brandhuber, P. Heslop, V. V. Khoze, B. Spence, and G. Travaglini, J. High Energy Phys. 05 (2009) 115.

[6] S. Caron-Huot, J. High Energy Phys. 12 (2011) 066.

[7] S. He, Z. Li, and C. Zhang, Phys. Rev. D 101, 061701(R) (2020).

[8] S. He, Z. Li, and C. Zhang, J. High Energy Phys. 03 (2021) 278.

[9] J. L. Bourjaily, A. J. McLeod, M. von Hippel, and M. Wilhelm, J. High Energy Phys. 08 (2018) 184.

[10] J. Henn, E. Herrmann, and J. Parra-Martinez, J. High Energy Phys. 10 (2018) 059.
[11] E. Herrmann and J. Parra-Martinez, J. High Energy Phys. 02 (2020) 099.

[12] L. F. Alday and J. M. Maldacena, J. High Energy Phys. 06 (2007) 064; L. F. Alday and J. Maldacena, J. High Energy Phys. 11 (2007) 068; 11 (2009) 082;

[13] A. Brandhuber, P. Heslop, and G. Travaglini, Nucl. Phys. B794, 231 (2008).

[14] J. Drummond, G. Korchemsky, and E. Sokatchev, Nucl. Phys. B795, 385 (2008); J. Drummond, J. Henn, G. Korchemsky, and E. Sokatchev, Nucl. Phys. B795, 52 (2008); Phys. Lett. B 662, 456 (2008); Nucl. Phys. B815, 142 (2009); Z. Bern, L. J. Dixon, D. A. Kosower, R. Roiban, M. Spradlin, C. Vergu, and A. Volovich, Phys. Rev. D 78, 045007 (2008).

[15] S. Caron-Huot, J. High Energy Phys. 07 (2011) 058.

[16] L. Mason and D. Skinner, J. High Energy Phys. 12 (2010) 018.

[17] N. Beisert et al., Lett. Math. Phys. 99, 3 (2012).

[18] L. F. Alday, D. Gaiotto, J. Maldacena, A. Sever, and P. Vieira, J. High Energy Phys. 04 (2011) 088.

[19] B. Basso, A. Sever, and P. Vieira, Phys. Rev. Lett. 111, 091602 (2013).

[20] S. Caron-Huot and S. He, J. High Energy Phys. 07 (2012) 174.

[21] Similar ideas have been used in [6] which motivated our investigations; they have also been explored [13,39].

[22] The $d \log$ representation plays an important role in the study of Feynman integrals, but our WL-based form differs from those studied before [2,11]; rather it takes a form very similar to those $\tau$ integrals in $\bar{Q}$ calculations.

[23] S. He, Z. Li, Y. Tang, and Q. Yang, J. High Energy Phys. 05 (2021) 052.

[24] N. Arkani-Hamed, J. L. Bourjaily, F. Cachazo, and J. Trnka, J. High Energy Phys. 06 (2012) 125.

[25] A. Hodges, J. High Energy Phys. 05 (2013) 135.

[26] J. M. Drummond, J. Henn, V. A. Smirnov, and E. Sokatchev, J. High Energy Phys. 01 (2007) 064; J. M. Drummond, J. Henn, G. P. Korchemsky, and E. Sokatchev, Nucl. Phys. B828, 317 (2010); G. P. Korchemsky and E. Sokatchev, Nucl. Phys. B839, 377 (2010).

[27] L. J. Dixon, J. M. Drummond, and J. M. Henn, J. High Energy Phys. 01 (2012) 024.

[28] For two-dimensional kinematics, $n=8$ double pentagons have been evaluated analytically in [40]; see [41] and [42] for analytic results of two-loop and three-loop amplitudes in two-dimensional kinematics.

[29] J. L. Bourjaily, A. J. McLeod, C. Vergu, M. Volk, M. Von Hippel, and M. Wilhelm, J. High Energy Phys. 02 (2020) 025.

[30] A. B. Goncharov, M. Spradlin, C. Vergu, and A. Volovich, Phys. Rev. Lett. 105, 151605 (2010); C. Duhr, H. Gangl, and J. R. Rhodes, J. High Energy Phys. 10 (2012) 075.

[31] It has been known since [43] that any one-loop integral in $\mathcal{N}=4 \mathrm{SYM}$ can be expanded into a basis of box integrals, where the coefficients are given by generalized unitarity cut, or one-loop leading singularities.

[32] Note that the loop momentum can be represented by two twistors on the line, $\ell \sim(A B)$, and the numerator can be written as $\langle\ell \bar{i} \cap \bar{j}\rangle=\langle A i-1 i i+1\rangle\langle B j-1 j j+1\rangle-$ $(A \leftrightarrow B)$; see [24] for more details. 
[33] D. Chicherin, S. Derkachov, and A. Isaev, J. High Energy Phys. 04 (2013) 020.

[34] N. Arkani-Hamed and E. Y. Yuan, arXiv:1712.09991.

[35] Throughout the Letter, any relabeling acts on external legs/momentum twistors, rather than on dual points. We denote the 15 residues of the hexagon (2-forms) by the two propagators that are not cut in each quadruple cut, e.g., $[x, y]$ denotes the residue with $x_{k}, x_{k+1}, x_{l}, x_{l+1}$ cut; by global residue theorem, only five of them are independent, which we choose to be $[x, y],\left[x, x_{k}\right], \ldots,\left[x, x_{l+1}\right]$.

[36] We still need to perform "rationalization" and encounter spurious square roots of kinematics from $w$, but they all nicely cancel in the final answer as expected.

[37] Note that when converting $d \log$ 's of $\tau^{\prime}$ into those of $z\left(\tau^{\prime}\right)$, we omitted "constants" that are independent of $\tau^{\prime}$; it is crucial to also drop the same constants for $d \log$ 's in the rational part since one can only ignore such constants for the entire, integrable symbol.
[38] See Supplemental Material at http://link.aps.org/supplemental/ 10.1103/PhysRevLett.126.231601 for a Mathematica readable expression of the symbol for $I_{\mathrm{dp}}(1,4,7,10)$ and w3symbolofdp.pdf for the expression of $R_{j-1 j}^{i}$ and $M_{j-1 j}^{i k l}$.

[39] C. Anastasiou and A. Banfi, J. High Energy Phys. 02 (2011) 064.

[40] L. F. Alday, J. High Energy Phys. 07 (2011) 080.

[41] P. Heslop and V. V. Khoze, J. High Energy Phys. 11 (2010) 035.

[42] S. Caron-Huot and S. He, J. High Energy Phys. 08 (2013) 101.

[43] Z. Bern, L. J. Dixon, and D. A. Kosower, Phys. Lett. B 302, 299 (1993); 318, 649(E) (1993); Nucl. Phys. B412, 751 (1994).

Correction: A support statement in the Acknowledgments section for the first author contained an incorrect grant number and has been fixed. 